\title{
Composition chimique et activité antibactérienne de l'huile essentielle de Lavandula officinalis cultivées dans la région de Skikda - Algérie.
}

\author{
Chemical composition and antibacterial activity of essential oil of \\ Lavandula officinalis grown in the region of Skikda - Algeria. \\ Manuscrit reçu le 26 avril 2017 et accepté le 22 juin 2017

\section{Hicham BOUGHENDJIOUA} \\ Département des sciences naturelles, l'école normale supérieure d'enseignement \\ technologique de Skikda (ENSET), 21000, Algérie. \\ Adresse électronique pour la correspondance : boughendjioua.hicham@yahoo.com
}

\section{Résumé :}

Cette étude a pour objectif de déterminer la composition chimique et d'évaluer l'activité antibactérienne de l'huile essentielle des sommités fleuries sèches de la lavande (Lavandula officinalis). L'analyse de l'huile essentielle par CG/SM a permis d'identifier 20 composés terpéniques dont les principaux sont : Linalyl acétate (32,98\%), Linalool $(28,92 \%)$, $\beta$ caryophyllène (4.62\%), Acétate de lavandulyle (4.52\%), Z- $\beta$-ocimène $(4.44 \%)$, terpinène-4ol $(4,32 \%)$, E- $\beta$-ocimène $(3.09 \%)$ et $\beta$-farnésène $(2.73 \%)$. L'évaluation de l'activité antibactérienne in vitro sur trois cocci Gram+ : streptocoque $\beta$-hémolytique du groupe A, Staphylococcus aureus et Staphylococcus epidermidis, souvent cause de la majorité des infections cutanées ont révélé un pouvoir antibactérien.

Mots clés : Huile essentielle, Lavandula officinalis, CG/SM, activité antibactérienne.

\section{Abstract:}

This study aims to determine the chemical composition and evaluate the antibacterial activity of the essential oil of flowers dry lavender buds (Lavandula officinalis). The analysis of the essential oil by GC / MS identified 20 terpenic compounds, the main ones: Linalyl acetate $(32,98 \%)$, Linalool $(28,92 \%), \beta$-caryophyllene $(4.62 \%)$, Lavandulyl acetate $(4.52 \%), Z-\beta-$ ocimene $(4.44 \%)$, terpinene-4-ol $(4,32 \%), \quad$ E- $\beta$-ocimene $(3.09 \%)$ and $\beta$-farnesene $(2.73 \%)$. The evaluation of the antibacterial activity in vitro on three Gram + cocci: $\beta$ hemolytic streptococcus group A, Staphylococcus aureus and Staphylococcus epidermidis often caused the majority of skin infections showed an antibacterial power.

Keywords: Essential oil, Lavandula officinalis, GC/MS, antibacterial activity.

\section{Introduction :}

Les infections microbiennes restent des affections graves et leur fréquence a augmenté de façon considérable au cours des dernières années en raison principalement du nombre accru de patients immunodéprimés et d'interventions médicochirurgicales invasives (Hulin et al., 2005). D'un autre côté, l'usage extensif des agents antibactériens et antifongiques chimiques dans la médication humaine ainsi que dans les élevages animaux conduit à la sélection de 
souches microbiennes résistantes, d'où l'importance d'orienter les recherches vers de nouvelles voies et surtout vers les végétaux qui ont toujours constitué une source d'inspiration de nouveaux médicaments. Ainsi, les huiles essentielles commencent à avoir beaucoup d'intérêts comme source potentielle de molécules naturelles bioactives. Elles font l'objet de multiples études pour leur éventuelle utilisation comme alternative dans le traitement des maladies infectieuses. Le traitement des mycoses cutanées bénéficie actuellement de nombreux antifongiques actifs et efficaces. Malgré cela, le problème des résistances de plus en plus nombreuses à un ou plusieurs antifongiques persiste encore. Par ailleurs, le problème des récidives n'est pas résolu et on sait qu'il n'y a pas d'immunité vis-à-vis des champignons, mais plutôt d'un terrain favorable aux mycoses. Ce problème de mycoses à répétitions a été soulevé et il semblerait s'agir beaucoup plus d'une absence d'éradication du germe que d'une infestation (Ouraïni et al., 2007).

La Lavande est un sous-arbrisseau de la famille des Labiées, Le genre se compose d'environ 28 espèces, qui sont dans la plupart d'origine méditerranéenne. Ce sous-arbrisseau est à tige et feuilles persistantes, il peut atteindre une longueur de 1 mètre, étroit vert pâle, s'étend du gris bleuâtre profond au vert à brun pâle, fleurs de couleur bleu - violet. D'autres variétés sont à fleurs blanches et roses (Chu et Kemper, 2001 ; Allaby, 1992). L'ensemble de la plante est très aromatique comprenant fleurs et feuilles La Lavande est employée comme expectorant, antispasmodique, désinfectant des plaies, contre les problèmes dermiques, possède des propriétés antimicrobiennes et anti-carcinogènes, sédatif, antidépresseur, antioxydant, antiinflammatoire et insecticide. (Chu et Kemper, 2001 ; Gören et al., 2002). Notre objectif dans ce travail est d'étudier l'activité antibactérienne de l'huile essentielle de la Lavande sur la croissance de trois souches bactériennes qui sont à l'origine de plusieurs infections cutanées.

\section{Matériel et méthodes}

\subsection{Matériel végétal}

Les échantillons de La Lavandula officinalis provient de la région d'Azzaba willaya de Skikda (Algérie), la récolte a été réalisée au mois de Mai 2013. Les sommités fleuries récoltées sont séchées à l'abri de la lumière et à température ambiante pendant 10 jours.

\subsection{Extraction de l'huile essentielle}

L'extraction de l'huile essentielle a été effectuée par hydrodistillation dans un appareil de type Clevenger (Clevenger, 1928) où l'on place $100 \mathrm{~g}$ des sommités fleuries séchées avec un litre d'eau distillée. L'huile essentielle obtenue est stockée au réfrigérateur dans un flacon en verre brun fermé hermétiquement à $4{ }^{\circ} \mathrm{C}$ et à l'ombre en présence de sulfate de sodium anhydre.

\subsection{Détermination de la composition chimique de l'huile essentielle par CG/SM}

La composition chimique de l'huile essentielle a été déterminée au niveau du laboratoire central de la police scientifique de Alger à l'aide d'un chromatographe en phase gazeuse couplé à un spectromètre de masse (CG/SM) de type Perkin Elmer modèle Clarus 500, équipé d'une colonne capillaire PE, Elite série $5 \%$ phenyl dimethylpolysiloxanne (30 m x 0,25 mm) avec une épaisseur du film de $0,25 \mu \mathrm{m}$, d'un injecteur split - splitless réglé à $250^{\circ} \mathrm{C}$. Le mode d'injection est split (rapport de fuite : 1/50, débit : $66 \mathrm{ml} / \mathrm{min}$ ). Les échantillons sont dilués dans le méthanol $(1 / 20 \mathrm{v} / \mathrm{v}), 2 \mu 1$ est injecté manuellement. Le gaz utilisé est l'Hélium avec un débit de $1 \mathrm{ml} / \mathrm{min}$. La température de la colonne est programmée de 60 à $275{ }^{\circ} \mathrm{C}$. La 
fragmentation est effectuée par impact électronique sous un champ de $70 \mathrm{eV}$, avec un balayage de 80 - 600 uma, un analyseur quadripôle et un délai de solvant : $5.90 \mathrm{mn}$. L'identification des constituants a été réalisée en se basant sur leurs indices de Kováts (IK). L'appareil est relié à un système informatique gérant une bibliothèque de spectre de masse NIST 98.

\section{4. Évaluation de l'activité antibactérienne}

Le test de l'activité antibactérienne de l'huile essentielle a été réalisé par la méthode de diffusion sur milieu gélosé ou aromatogramme (NCCLS,1997). L'aromatogramme est une méthode inspirée de l'antibiogramme, il permet de déterminer l'activité inhibitrice de l'huile essentielle par mesure du diamètre d'inhibition, autour d'un disque imprégné de celle-ci, ou d'un produit à base d'huile essentielle (Vincent,1991). Trois concentrations d'huile essentielle ont été testées, l'extrait naturel brut ainsi que deux dilutions au un-demi et au un-quart de celui-ci. Ces huiles ont été diluées dans le DMSO (Diméthyle sulfoxide).

Le choix des bactéries a été porté sur trois souches fréquentes en pathologie humaine (Infections cutanées) et pour leur résistance aux antibiotiques. Les souches bactériennes sont : streptocoque $\beta$-hémolytique du groupe A, Staphylococcus aureus et Staphylococcus epidermidis. Ces souches nous ont été fournies aimablement par le responsable de laboratoire de Microbiologie de centre hospitaliers Universitaires (CHU) Ibn Rochd d'Annaba, (Algérie). Un disque de $6 \mathrm{~mm}$ de diamètre en papier Whatman stérile est imprégné de l'huile essentielle de Lavandula officinalis puis déposé au milieu d'une boite de Petri de $90 \mathrm{~mm}$ de diamètre contenant un milieu gélosé « Mueller Hinton » de $4 \mathrm{~mm}$ d'épaisseur, préalablement ensemencé par écouvillonnage avec l'inoculum. Ce dernier est préparé à une concentration de 0,5 Marc Ferland, de 106 à 108CFU.mL-1 pour chaque souche. Un disque imprégné avec le DMSO (Diméthyle sulfoxide) est appliqué dans les boites témoins, trois répétitions sont réalisées pour chaque essai. Les boites de Petri sont incubées pendant $24 \mathrm{~h}$ à $37^{\circ} \mathrm{C}$, la lecture s'effectue par la mesure du diamètre de la zone d'inhibition, qui se traduit par un halo translucide autour de chaque disque ; la présence ou l'absence d'un halo expliquerait la sensibilité ou la résistance des germes vis-à-vis des extraits testés ; selon une échelle de notation symbolique allant de - à +++ et dont la lecture d'après (Meena et Sethi, 1994 ; Jeovetz et al., 2000) se fait comme suit :

- $\varnothing<10 \mathrm{~mm}$ : huile essentielle (HE) sans action inhibitrice (-)

- $16>\varnothing \geq 10 \mathrm{~mm}:$ HE à une action inhibitrice intermédiaire $(+)$

- $25>\varnothing \geq 16 \mathrm{~mm}$ : HE à une action inhibitrice importante $(++)$

- $\varnothing \geq 25 \mathrm{~mm}:$ HE à une action inhibitrice très efficace $(+++)$

\section{Résultats et discussion}

\subsection{Rendement}

Les résultats obtenus indiquent que le rendement d'extraction de l'huile essentielle par hydrodistillation est de $1,50 \pm 0,2 \%$. La cinétique d'extraction a montré que la quasi-totalité de l'huile essentielle est extrait au bout des 90 premières minutes.

Les résultats obtenus par Laib et Barbat (2011) et Mohammadi et al., (2011) indiquent que les fleurs sèches de la lavande provenant de deux régions d'Algérie présentent des teneurs en huile essentielle respectivement $1.36 \%$ et $2.01 \%$. 
Bouguerra et Zeghou (2009) ont trouvé que les fleurs de Lavandula officinalis collecté du même lieu ont présenté un rendement de $3.41 \%$.

De même, les résultats obtenus par Sidi Boulenouar et Ziane (2003) indiquent que les fleurs sèches de la lavande provenant de la région d'Ouchba et Zarifet ont donné des teneurs en huile essentielle équivalentes respectivement à $0.94 \%$ et $0.70 \%$.

Ces variations de teneurs peuvent être dues à plusieurs facteurs notamment le degré de maturité des fleurs de Lavandula officinalis, l'interaction avec l'environnement (type de climat, sol), le moment de la récolte et la méthode d'extraction (Botton et al., 1990).

\subsection{Composition chimique}

L'analyse qualitative et quantitative par (GC/SM) de l'huile essentielle a permis d'identifier 20 composés qui représentent un total de 91,84\% (Tableau 01). L'essence de Lavandula officinalis est constituée principalement de : Linalyl acétate (32,98\%), Linalool $(28,92 \%)$, $\beta$ caryophyllène (4.62 \%), Acétate de lavandulyle (4.52\%), Z- $\beta$-ocimène $(4.44 \%)$,terpinène-4ol $(4,32 \%)$, E- $\beta$-ocimène $(3.09 \%), \beta$-farnésène $(2.73 \%)$ totalisant environ 85,62\%.

Des études réalisées également en Algérie par Laib et Barbat (2011) pour la région de Constantine ont donné : Linalyl acétate (15.26\%), Linalool (10.68\%), 1,8- cineole (10.25\%), $\gamma$-terpinene (11.2 $\%$ ) et camphor (11.25\%). Nos résultats et ceux de Laib et Barbat (2011) sont différents de ceux indiqués par certains auteurs. Kulevanova et al. (2000) ont analysé la composition chimique de l'huile essentielle des fleurs de Lavandula officinalis collectées de la montagne de Kozjak (Macedonia), ils ont trouvé 32 constituants avec une prédominance de linalool $(25,7 \%)$, linalyl acétate $(23,2 \%)$ et lavandulyl acetate $(12,4 \%)$ avec une dominance des composants mono terpéniques et la présence des hydrocarbures sesqueterpéniques et ses dérivés oxygénés. Verma et al. (2009) ont étudié la composition de l'huile essentielle des fleurs de Lavandula officinalis cultivées à Uttarakand (Inde), ils ont identifié 37 composés monoterpéniques. Les composés majeurs étaient : linalyl acétate $(47,56 \%)$, linalool $(28,06 \%)$, lavandulyl acétate $(4,34 \%)$ et $\alpha$ - terpineol $(3,7 \%)$.

Sun Kim et Sun Lee (2002) ont comparé la composition chimique des huiles essentielles de Lavandula officinalis obtenues par différentes méthodes d'extraction. Ils ont trouvé que le linalyl acétate $(35,44 \%)$ et le linalool $(18,70 \%)$ sont prédominants dans les huiles essentielles obtenues par distillation à la vapeur tandis que leurs valeurs étaient respectivement de 2,63 et 4,04\% dans le cas d'extraction par solvants ; 36,80 et 43,47\% dans le cas d'extraction par microonde.

D'après ces résultats, on remarque que la composition chimique de l'huile essentielle de l'espèce Lavandula officinalis cultivée à Constantine est différente de celles obtenues dans de nombreux travaux sur la même espèce, avec une prédominance des composés mono terpéniques dans la plupart des cas, mais à des proportions différentes.

Cette différence de composition est due probablement à diverses conditions notamment l'environnement, le génotype, l'origine géographique, la période de récolte, le lieu de séchage, la température et la durée de séchage, les parasites et la méthode d'extraction (Svoboda et Hampson, 1999). 
Tableau 01 : Composition chimique de l'huile essentielle de Lavandula officinalis

\begin{tabular}{|c|c|c|c|}
\hline $\mathbf{N}^{\mathbf{o}}$ & Composé & Indice de Kovats & $(\mathrm{m} / \mathrm{m}) \%$ \\
\hline 01 & Octanone-3 & 1140 & $0.72 \%$ \\
\hline 02 & Acétate d'octène-3-yle & 1165 & $0.65 \%$ \\
\hline 03 & $\alpha$-terpinéol & 1169 & $0,90 \%$ \\
\hline 04 & $\beta$-phellandrène & 1177 & $0.12 \%$ \\
\hline 05 & Acétate de néryle & 1189 & $0,32 \%$ \\
\hline 06 & Linalol & 1202 & $28,92 \%$ \\
\hline 07 & Camphre & 1212 & $0.85 \%$ \\
\hline 08 & terpinène-4-ol & 1223 & $4,32 \%$ \\
\hline 09 & Nérol & 1250 & $0,20 \%$ \\
\hline 10 & Acétate de lavandulyle & 1269 & $4.52 \%$ \\
\hline 11 & Acétate de géranyle & 1287 & $0,60 \%$ \\
\hline 12 & Myrcène & 1290 & $0.46 \%$ \\
\hline 13 & $\beta$-caryophyllène & 1298 & $4.62 \%$ \\
\hline 14 & Germacrène & 1362 & $0.27 \%$ \\
\hline 15 & E- $\beta$-ocimène & 1374 & $3.09 \%$ \\
\hline 16 & Z- $\beta$-ocimène & 1391 & $4.44 \%$ \\
\hline 17 & Cryptone & 1411 & $0,35 \%$ \\
\hline 18 & $\beta$-farnésène & 1418 & $2.73 \%$ \\
\hline 19 & Lavandulol & 1439 & $0,78 \%$ \\
\hline 20 & Acétate de linalyle & 1454 & $32,98 \%$ \\
\hline & Total & & $91,84 \%$ \\
\hline
\end{tabular}

\subsection{Activité antibactérienne}

Il s'agit de déterminer le diamètre d'inhibition selon la méthode de diffusion sur les disques stériles. Nous avons testé l'effet de l'huile essentielle brute de la lavande, sur trois souches bactériennes (streptocoque $\beta$-hémolytique $d u$ groupe A, Staphylococcus aureus, Staphylococcus epidermidis). Les résultats expérimentaux présentés dans le Tableau 02 montrent que l'huile essentielle de la lavande extraite présente une activité importante. Plusieurs travaux (Xianfei et al., 2007 ; Sandri et al., 2007 ; Zarai et al., 2011 ; Al-Bayati, 2008) ont rapporté que les bactéries Gram (+) sont plus susceptibles aux huiles essentielles que les bactéries Gram (-) attribuée à la présence d'une membrane externe, imperméable aux composés hydrophobes grâce à son revêtement lipopoly saccharide.

L'absence de cette barrière, chez les bactéries Gram (+) permet le contact direct des constituants hydrophobes de l'huile essentielle avec la bicouche phospholipidique de la membrane cellulaire, provoquant ainsi soit, une augmentation de la perméabilité des ions et la fuite des constituants intracellulaires vitaux, soit une déficience au niveau du système enzymatique (Sandri et al., 2007 ; Zarai et al., 2011 ; Al-Bayati, 2008 ; Randrianarivelo et al., 2009).

Nos résultats sont en accord avec ceux de Chahboun et al. (2015) et Jianu et al. (2013) qui ont observé une sensibilité importante chez Staphylococcus aureus vis-à-vis l'huile essentielle de la lavande.

Par ailleurs, l'activité antibactérienne de notre huile essentielle peut aussi, être attribuée au phénomène de synergie entre tous les constituants volatiles ; les interactions synergiques 
entres les différents composés peuvent être à l'origine d'une activité beaucoup plus prononcée que celle prévisible pour les composés majoritaires. Ceci est confirmé par plusieurs études (Al-Bayati, 2008 ; Randrianarivelo et al., 2009 ; Hmamouchi et al., 2001).

Tableau 02 : Activité antibactérienne de l'huile essentielle de lavandula officinalis.

\begin{tabular}{|l|c|c|c|c|c|c|}
\hline \multicolumn{1}{|c|}{ Souches } & HE & HE' & HE' & PIP & CAZ & OX \\
\hline \hline streptocoque $\beta$-hémolytique du groupe A & +++ & ++ & + & S & R & nd \\
\hline Staphylococcus aureus & +++ & ++ & + & $\mathrm{R}$ & $\mathrm{R}$ & $\mathrm{nd}$ \\
\hline Staphylococcus epidermidis & +++ & ++ & + & $\mathrm{R}$ & $\mathrm{R}$ & $\mathrm{nd}$ \\
\hline
\end{tabular}

HE: huile essentielle brute; HE': HE diluée à 1/2 (v/v); HE': HE diluée à 1/4 (v/v); CAZ: ceptazidine; PIP: piperacilline; OX: oxacilline; R: résistante; $S$ : sensible; nd: non déterminée.

\section{Conclusion}

Le présent travail a visé la détermination du rendement, la composition chimique et les propriétés antibactériennes de l'huile essentielle de la lavande cultivée dans de la région d'Azzaba willaya Skikda.

Les résultats obtenus indiquent que le rendement d'extraction de l'huile essentielle par hydro

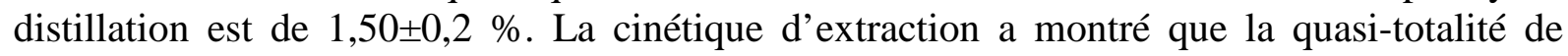
l'huile essentielle est extrait au bout des 90 premières minutes.

L'analyse de la composition chimique par analysée par GC/MS a permis d'identifier 20 composés terpéniques dont les principaux sont: Linalyl acétate $(32,98 \%)$, Linalool $(28,92 \%)$, $\beta$-caryophyllène (4.62\%), Acétate de lavandulyle (4.52\%), Z- $\beta$-ocimène $(4.44 \%)$, terpinène4-ol $(4,32 \%)$, E- $\beta$-ocimène (3.09\%) et $\beta$-farnésène $(2.73 \%)$.

L'huile essentielle de Lavandula officinalis est un antibactérien naturel très efficaces et peuvent être une source très importante de constituants phytopharmaceutiques utilisés pour éradiquer les infections dermiques.

\section{Références :}

Al-Bayati A.F. (2008). Synergistic antibacterial activity between Thymus vulgaris and Pimpinellaanisum essential oils and methanol extracts, Ethnopharmacology, Vol. 116, 403406.

Allaby M. (1992). The Concise Oxford Dictionary of Botany, Oxford University Press. y First edition., New York.

Botton B., Bertron A., Fevere M., Gauthier S., Guph D., Plarpent J., Reymond P., Sanglier J.J, Vaysser Y et Veau S. (1990). Moisissures utiles et nuisibles importance industrielle. Ed : Masson collection biotechnologies, Paris.

Bouguerra A et Zeghou K. (2009). Etude des activités antioxydante et antibactérienne de l'huile essentielle des fleurs sèches de Lavandula stoechas L. Mémoire d'ingénieur INATAA.

Chahboun N., Esmail A., Abed1 H., Barrahi M., Amiyare R., Berrabeh M., Oudda H et Ouhssine M. Evaluation de l'activité bactériostatique d'huile essentielle de la Lavandula officinalis vis-à-vis des souches d'origine clinique résistantes aux antibiotiques, (2015), 1191. 
Chu C.J and Kemper K.J. (2001). Lavender (Lavandula spp.). Longwood Herbal Task Force. Boston, (2001), p 32.

Clevenger J. F. (1928). Apparatus for volatile oil determination: description of New Type Clevenger. Am. Perf. Ess. Oil Review, 467-503.

Gören A.C., Topçu G., Bilsela G., Bilsela M., Aydoğmus Z et Pezzuto J.M.Z. (2002). Naturforsch, 57c, 797-800.

Hmamouchi M., Hamamouchi J., Zouhdi M et Bessiere J.M. (2001). Chemical and antimicrobial properties of essential oils of five Moroccan Pinaceae. Essential Oil Research, Vol.13, 298-302.

Hulin A., Deguillaume A.M., Bretagne S et Bézie Y. (2005). Bon usage des antifongiques dans le traitement des candidoses et aspergilloses invasives - J Pharm Clin, Vol.24, $\mathrm{N}^{\circ} 3,125$ 38 .

Jeovetz L., Buchbauer G., Stoyanova A and Metodiev S. (2000). Seasonal depending variations of the composition and biological activities of Douglas fir (Pseudo tsugamenzlesil) essential oils from Bulgaria, Scientia Pharmazeutica, Vol. 66, 323-328.

Jianu C., Pop G., Gruia A.T and Horhat F.G. (2013). Chemical Composition and Antimicrobial Activity of Essential Oils of Lavender (Lavandula angustifolia) and Lavandin (Lavandula x intermedia) Grown in Western Romania, Int. J. Agric. Biol, Vol. 15, No 4, 772776.

Kulevanova S., Stetkov G and Ristic M. (2000). Examination of and essential oils of Lavandula officinalis grown on mountain KOZJAK (MACEDONIA). Bulletin of the Chemists and Technologists of Macedonia, 19 (2), 165-169.

Laib I et Barbat M. (2011). Composition chimique et activité antioxydante de l'huile essentielle des fleurs sèches de Lavandula officinalis, Revue de génie industriel, 6, 46-54.

Meena M.R and Sethi V. (1994). Antimicrobial activity of the essential oils from spices, Food Science and Technology, Vol. 31, 68-70.

Mohammedi Z et Atik F. (2011). Nature \& Technologie, 6, 34-39.

NCCLS (National Committee for Clinical Laboratory Standards). (1997). Performance standards for antimicrobial disk susceptibility test. Sixth Ed. Approved Standard M2-A6, Wayne, PA.

Ouraïni D., Agoumi A., Alaoui M.I., Alaoui K., Cherrah Y., Alaoui M.A et Belabbas M.A. (2007). Activité antifongique de l'acide oléique et des huiles essentielles de Thymus saturejoides L. et de Mentha pulegium L., comparé aux antifongiques dans les dermatoses mycosiques-Phytothérapie, Vol.1, 6-14.

Randrianarivelo R., Sarter S., Odoux E., Brat P., Lebrun M., Romestand B., Menut C and Andrianoelisoa H.S, Raherimandimby M. and Danthu P. (2009). Composition and antimicrobial activity of essential oils of Cinnamosma fragrans, Food Chemistry, Vol. 114, 680-684. 
Sandri I.G., Zacaria J., Fracaro F., Delamare A.P.L and Echeverrigaray S. (2007). Antimicrobial activity of the essential oils of Brazilian species of the genus Cunilaagainst food borne pathogens and spoiling bacteria, Food Chemistry, Vol. 103, 823-828.

Sidi Boulenouar K et Ziane A. (2003). Etude phytochimique des huiles essentielles de Lavandula stoechas L. de la région de Tlemcen. Mémoire pour l'obtention du diplôme d'études supérieur en biologie. Option : Biochimie. Faculté des Sciences. Univ. ABB. Tlemcen, p 54.

Sun Kim N and Sun Lee D.S. (2002). Comparison of different extraction methods for the analysis of fragrances from Lavandula species by gas chromatography-mass spectrometry. Journal of Chromatography, 982, 31-47.

Svoboda K.P and Hampson J.B. (1999). Bioactivity of essential oils of selected temperate aromatic plants: antibacterial, antioxidant, anti-inflammatory and other related pharmacological activities. Plant Biology Department, SAC Auchincruive, Ayr, Scotland, UK., KA6 5HW.

Verma R.S., Laiq U., Rahman S., Chandan S., Chanotiya K., Rajesh K., Chauhan A., Yadav A and Singh A. (2009). Essential oil composition of Lavandula officinalis cultivated in the mid hills of Uttarakhand, India. J. Serb. Chem. Soc, 75 (3), 343-348.

Vincent M.C. (1991). L’aromatogramme. Encyclopédie de médecine naturelle, phytothérapie, aromathérapie. 4, Paris 6.

Xianfei X., Xiaoqiang C and Shunying Z.G. (2007). Chemical composition and antimicrobial activity of essential oils of Chaenomelesspeciosa from China, Food Chemistry, Vol. 100, 1312-1315.

Zarai Z., Kadri A., Ben Chobba I., Ben Mansour R., Bekir A., Mejdoub A and Gharsallah N. (2011). The in-vitro evaluation of antibacterial, antifungal and cytotoxic properties of Marrubium vulgare L. essential oil grown in Tunisia, Lipids in Health and Disease, Vol.10, p 161. 